\title{
INTERPRETATION OF THE RAPID INTRAVENOUS GLUCOSE TOLERANCE TEST IN NORMAL INDIVIDUALS AND IN MILD DIABETES MELLITUS ${ }^{1}$
}

\author{
By DONALD S. AMATUZIO,² FRANCIS L. STUTZMAN, MURIEL J. VANDERBILT, \\ AND SAMUEL NESBITT
}

(From the Veterans Administration Hospital and the Department of Medicine, University of Minnesota Medical School, Minneapolis, Minn.)

(Submitted for publication November 7, 1952; accepted January 21, 1953)

The glucose tolerance test is an important diagnostic procedure in metabolic diseases. As the test is currently employed, glucose is administered either orally or intravenously and there is no uniform interpretation of the results.

A study of the rapid glucose tolerance test is found in a review by Tunbridge and Allibone (1). The present study was undertaken to test the validity of the concept that the rapid intravenous glucose tolerance test might properly and readily be interpreted by following the rate of disappearance of glucose from the blood. It was our hope also to evolve a simple test for routine use. The uniformity of the single injection method was studied as a means of separating the normal from the known mild diabetic patient.

\section{MATERIAL AND METHODS}

Twenty-five grams of glucose as a 30 per cent solution in distilled water were administered intravenously within four minutes. A fasting blood specimen was obtained, and then at four minutes following the glucose administration and subsequently every eight minutes for 72 minutes. Blood was obtained from an ear lobe that was kept moist with a heparin sponge. Determination of blood glucose was done by the Horvath and Knehr modification (2) of the Folin-Malmros micromethod (3).

The control group consisted of 70 healthy men ranging in age from 25 to 50 years with no family history of diabetes mellitus. The test was repeated with 25 grams of glucose at intervals of from one to three months in 20 subjects, and with 35 grams of glucose in 13 who had been on an adequate carbohydrate intake. The abnormal group consisted of 26 patients with known mild diabetes mellitus controlled by diet alone (the majority had

1 Published with the approval of the Chief Medical Director. The statements and conclusions published by the authors are the result of their own study and do not necessarily reflect the opinion or policy of the Veterans Administration.

2 Present address: Lt. D. S. Amatuzio, MC, USNR (Staff), U. S. Naval Hospital, Oakland 14, California. normal fasting blood sugars) and 13 patients with severe diabetes mellitus. Diabetes mellitus had been diagnosed in these patients by clinical evaluation and the 100 grams standard oral glucose tolerance test.

\section{RESULTS}

The blood glucose values of the 25 grams rapid glucose tolerance test, expressed as mgm. per cent glucose excess, ${ }^{3}$ in 70 normal men with no family history of diabetes mellitus are given in Table $\mathrm{I}$. Similar blood (mgm. per cent) glucose excess values were found in 20 normal men who had a repeat 25 grams test one to three months later

${ }^{8}$ Milligram per cent glucose excess is defined as that amount of glucose in excess of the fasting blood glucose in mgm. per cent.

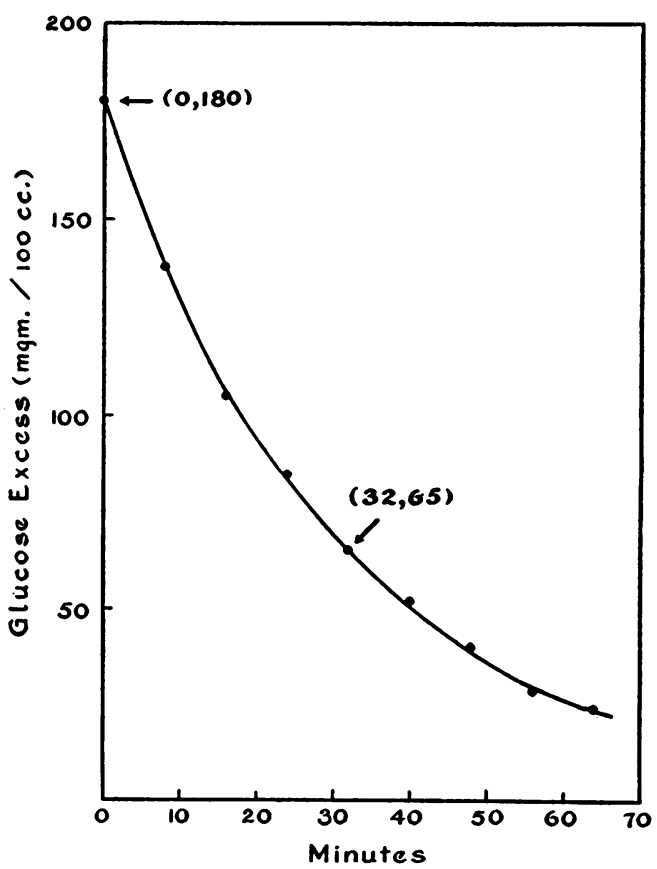

Fig. 1. Glucose Disappearance Plotted on Coordnate Graph Paper-Patient W. B. 
and who had been on an adequate carbohydrate intake (Table II). A comparison of the 25 grams to the $\mathbf{3 5}$ grams test showed different $\mathrm{mgm}$. per cent glucose excess values at determined times in 13 normal men who had been on an adequate carbohydrate intake (Table IV). In 39 patients with diabetes mellitus (26 were mild and 13 were severe diabetics) the 25 grams glucose tolerance test gave higher mgm. per cent glucose excess values (Table $\mathrm{V}$ ).

The rate of disappearance of glucose was found to be apparently proportional to the concentration if the glucose excess in mgm. per $100 \mathrm{cc}$. is plotted against time using the four minute glucose value after injection as that value at time zero (Figure 1). This is best represented by an equation of a monomolecular reaction:

$$
C(t)=C(o) e^{-k t}
$$

where

$$
C(t)=\text { the glucose excess in mgm. per } 100 \mathrm{cc} \text {. }
$$
at any time, $t$,

$C(0)=$ the glucose excess in mgm. per $100 \mathrm{cc}$. at time zero,

and

$$
\begin{aligned}
& \mathrm{k}=\text { the disappearance rate of glucose from } \\
& \text { the blood. }
\end{aligned}
$$

\begin{tabular}{|c|c|c|c|c|c|c|c|c|c|c|}
\hline \multirow[b]{2}{*}{ Patient } & \multirow[b]{2}{*}{$\begin{array}{l}\text { Fasting } \\
\text { glucose }\end{array}$} & \multicolumn{8}{|c|}{ Glucose excess (mgm.\%) } & \multirow{2}{*}{$\begin{array}{c}\text { Rate of } \\
\text { removal } \\
(\% / \mathrm{min} .)\end{array}$} \\
\hline & & $\mathbf{0}$ & 8 & 16 & $\begin{array}{c}24 \\
\text { (time in }\end{array}$ & $\begin{array}{c}32 \\
\text { tes) }\end{array}$ & 40 & 48 & 56 & \\
\hline $\begin{array}{l}\text { C. B. } \\
\text { P. D. } \\
\text { R. P. } \\
\text { S. B. } \\
\text { G. S. } \\
\text { P. W. } \\
\text { R. G. } \\
\text { J. D. } \\
\text { L. I. } \\
\text { P. K. }\end{array}$ & $\begin{array}{r}78 \\
88 \\
104 \\
105 \\
115 \\
93 \\
93 \\
93 \\
95 \\
90\end{array}$ & $\begin{array}{l}182 \\
207 \\
140 \\
151 \\
145 \\
215 \\
205 \\
169 \\
160 \\
225\end{array}$ & $\begin{array}{r}140 \\
142 \\
91 \\
95 \\
108 \\
155 \\
150 \\
122 \\
121 \\
173\end{array}$ & $\begin{array}{r}100 \\
109 \\
66 \\
80 \\
73 \\
107 \\
108 \\
94 \\
83 \\
133\end{array}$ & $\begin{array}{r}80 \\
77 \\
44 \\
60 \\
45 \\
82 \\
71 \\
65 \\
58 \\
110\end{array}$ & $\begin{array}{l}57 \\
65 \\
31 \\
47 \\
34 \\
39 \\
50 \\
50 \\
40 \\
85\end{array}$ & $\begin{array}{l}47 \\
38 \\
20 \\
33 \\
25 \\
40 \\
28 \\
41 \\
40 \\
77\end{array}$ & $\begin{array}{r}35 \\
27 \\
1 \\
10 \\
-6 \\
30 \\
15 \\
34 \\
23 \\
55\end{array}$ & $\begin{array}{r}17 \\
12 \\
1 \\
1 \\
-6 \\
-10 \\
-6 \\
15 \\
6 \\
30\end{array}$ & $\begin{array}{l}3.44 \\
4.30 \\
4.81 \\
3.75 \\
4.60 \\
3.98 \\
4.53 \\
3.51 \\
4.21 \\
3.05\end{array}$ \\
\hline $\begin{array}{l}\text { W. B. } \\
\text { A. K. } \\
\text { M. C. } \\
\text { D. C. } \\
\text { W. B. } \\
\text { C. C. } \\
\text { W. K. N. } \\
\text { V. C. } \\
\text { W. B. } \\
\text { R. C. }\end{array}$ & $\begin{array}{r}122 \\
115 \\
88 \\
91 \\
123 \\
88 \\
84 \\
83 \\
85 \\
97\end{array}$ & $\begin{array}{l}153 \\
195 \\
199 \\
211 \\
173 \\
195 \\
241 \\
183 \\
177 \\
183\end{array}$ & $\begin{array}{l}106 \\
141 \\
141 \\
157 \\
120 \\
134 \\
184 \\
124 \\
138 \\
141\end{array}$ & $\begin{array}{r}76 \\
100 \\
104 \\
119 \\
85 \\
100 \\
142 \\
95 \\
105 \\
109\end{array}$ & $\begin{array}{r}56 \\
73 \\
70 \\
105 \\
65 \\
72 \\
104 \\
67 \\
85 \\
82\end{array}$ & $\begin{array}{r}41 \\
52 \\
52 \\
103 \\
45 \\
52 \\
89 \\
51 \\
65 \\
65\end{array}$ & $\begin{array}{l}28 \\
30 \\
34 \\
72 \\
35 \\
28 \\
76 \\
42 \\
52 \\
53\end{array}$ & $\begin{array}{r}5 \\
10 \\
12 \\
57 \\
23 \\
23 \\
50 \\
29 \\
40 \\
41\end{array}$ & $\begin{array}{r}1 \\
-13 \\
-8 \\
41 \\
7 \\
16 \\
42 \\
19 \\
29 \\
26\end{array}$ & $\begin{array}{l}4.14 \\
4.53 \\
4.37 \\
3.00 \\
4.06 \\
4.06 \\
3.12 \\
3.90 \\
3.20 \\
3.20\end{array}$ \\
\hline $\begin{array}{l}\text { M. W. } \\
\text { W. W. } \\
\text { G. T. } \\
\text { J. J. } \\
\text { K. H. } \\
\text { E. M. } \\
\text { H. O. } \\
\text { E. R. } \\
\text { L. E. } \\
\text { C. L. }\end{array}$ & $\begin{array}{r}85 \\
98 \\
93 \\
95 \\
128 \\
100 \\
90 \\
80 \\
92 \\
78\end{array}$ & $\begin{array}{l}157 \\
192 \\
179 \\
182 \\
190 \\
140 \\
175 \\
191 \\
188 \\
197\end{array}$ & $\begin{array}{l}112 \\
139 \\
140 \\
152 \\
132 \\
116 \\
126 \\
165 \\
143 \\
132\end{array}$ & $\begin{array}{r}82 \\
107 \\
101 \\
98 \\
124 \\
86 \\
100 \\
118 \\
105 \\
114\end{array}$ & $\begin{array}{l}67 \\
87 \\
81 \\
81 \\
89 \\
69 \\
78 \\
95 \\
85 \\
73\end{array}$ & $\begin{array}{l}56 \\
64 \\
59 \\
70 \\
57 \\
50 \\
56 \\
75 \\
69 \\
55\end{array}$ & $\begin{array}{l}48 \\
50 \\
50 \\
50 \\
46 \\
38 \\
48 \\
58 \\
52 \\
43\end{array}$ & $\begin{array}{l}36 \\
25 \\
35 \\
38 \\
34 \\
30 \\
36 \\
48 \\
36 \\
28\end{array}$ & $\begin{array}{r}18 \\
9 \\
19 \\
17 \\
25 \\
8 \\
20 \\
36 \\
29 \\
22\end{array}$ & $\begin{array}{l}3.30 \\
3.44 \\
3.51 \\
3.08 \\
4.06 \\
3.43 \\
3.35 \\
3.32 \\
3.20 \\
3.90\end{array}$ \\
\hline $\begin{array}{l}\text { A. S. } \\
\text { F. Mc. } \\
\text { C. A. } \\
\text { B. H. } \\
\text { E. S. } \\
\text { S. R. } \\
\text { H. H. } \\
\text { K. N. } \\
\text { J. S. } \\
\text { B. D. }\end{array}$ & $\begin{array}{r}105 \\
92 \\
83 \\
81 \\
95 \\
88 \\
95 \\
97 \\
87 \\
97\end{array}$ & $\begin{array}{l}160 \\
183 \\
204 \\
167 \\
178 \\
150 \\
165 \\
158 \\
164 \\
167\end{array}$ & $\begin{array}{l}113 \\
125 \\
141 \\
115 \\
140 \\
111 \\
118 \\
131 \\
135 \\
116\end{array}$ & $\begin{array}{r}81 \\
91 \\
94 \\
85 \\
107 \\
92 \\
95 \\
106 \\
110 \\
88\end{array}$ & $\begin{array}{l}67 \\
62 \\
75 \\
55 \\
87 \\
72 \\
70 \\
86 \\
82 \\
71\end{array}$ & $\begin{array}{l}51 \\
41 \\
59 \\
29 \\
68 \\
59 \\
51 \\
73 \\
61 \\
52\end{array}$ & $\begin{array}{l}36 \\
26 \\
34 \\
29 \\
43 \\
46 \\
37 \\
49 \\
42 \\
28\end{array}$ & $\begin{array}{l}32 \\
18 \\
28 \\
21 \\
42 \\
13 \\
29 \\
36 \\
35 \\
28\end{array}$ & $\begin{array}{r}22 \\
8 \\
10 \\
-3 \\
23 \\
25 \\
11 \\
28 \\
23 \\
1\end{array}$ & $\begin{array}{l}3.49 \\
4.84 \\
4.16 \\
4.45 \\
3.04 \\
3.12 \\
3.67 \\
3.04 \\
3.82 \\
3.95\end{array}$ \\
\hline
\end{tabular}

TABLE I

The rapid intravenous glucose tolerance (25 grams) and its rate of removal in normal men 
TABLE I-Continued

\begin{tabular}{|c|c|c|c|c|c|c|c|c|c|c|}
\hline \multirow[b]{2}{*}{ Patient } & \multirow[b]{2}{*}{$\begin{array}{l}\text { Fasting } \\
\text { glucose }\end{array}$} & \multicolumn{8}{|c|}{ Glucose excess (mgm.\%) } & \multirow{2}{*}{$\begin{array}{l}\text { Rate of } \\
\text { removal } \\
(\% / \text { min.) }\end{array}$} \\
\hline & & $\mathbf{0}$ & 8 & 16 & (time in & $\begin{array}{l}32 \\
\text { tes) }\end{array}$ & 40 & 48 & 56 & \\
\hline $\begin{array}{l}\text { R. F. } \\
\text { T. R. } \\
\text { R. C. H. } \\
\text { F. R. } \\
\text { E. E. } \\
\text { D. R. U. } \\
\text { V. K. } \\
\text { S. L. } \\
\text { H. } \\
\text { R. A. J. }\end{array}$ & $\begin{array}{r}87 \\
82 \\
87 \\
91 \\
110 \\
102 \\
102 \\
93 \\
98 \\
82\end{array}$ & $\begin{array}{l}206 \\
188 \\
185 \\
230 \\
173 \\
161 \\
165 \\
137 \\
177 \\
156\end{array}$ & $\begin{array}{l}143 \\
133 \\
134 \\
150 \\
113 \\
123 \\
123 \\
113 \\
120 \\
114\end{array}$ & $\begin{array}{r}101 \\
96 \\
95 \\
124 \\
80 \\
100 \\
103 \\
98 \\
92 \\
88\end{array}$ & $\begin{array}{r}85 \\
68 \\
74 \\
114 \\
50 \\
78 \\
76 \\
72 \\
76 \\
74\end{array}$ & $\begin{array}{l}54 \\
44 \\
48 \\
84 \\
35 \\
56 \\
62 \\
55 \\
53 \\
51\end{array}$ & $\begin{array}{l}40 \\
35 \\
35 \\
67 \\
25 \\
38 \\
45 \\
42 \\
41 \\
40\end{array}$ & $\begin{array}{l}28 \\
20 \\
17 \\
57 \\
-3 \\
28 \\
33 \\
37 \\
27 \\
30\end{array}$ & $\begin{array}{r}10 \\
14 \\
5 \\
36 \\
-5 \\
13 \\
24 \\
25 \\
15 \\
13\end{array}$ & $\begin{array}{l}4.30 \\
4.10 \\
4.53 \\
3.35 \\
4.84 \\
3.98 \\
3.36 \\
3.36 \\
3.84 \\
3.46\end{array}$ \\
\hline $\begin{array}{l}\text { G. F. E. } \\
\text { E. G. } \\
\text { M. K. } \\
\text { A. Z. } \\
\text { I. P. } \\
\text { W. G. } \\
\text { W. M. } \\
\text { W. J. } \\
\text { E. G. } \\
\text { S. D. }\end{array}$ & $\begin{array}{l}95 \\
85 \\
95 \\
78 \\
84 \\
88 \\
98 \\
87 \\
86 \\
97\end{array}$ & $\begin{array}{l}138 \\
187 \\
153 \\
196 \\
180 \\
169 \\
174 \\
168 \\
175 \\
128\end{array}$ & $\begin{array}{r}99 \\
143 \\
119 \\
142 \\
144 \\
123 \\
133 \\
130 \\
133 \\
107\end{array}$ & $\begin{array}{r}77 \\
97 \\
100 \\
117 \\
112 \\
97 \\
118 \\
101 \\
107 \\
76\end{array}$ & $\begin{array}{r}57 \\
63 \\
82 \\
90 \\
82 \\
72 \\
102 \\
76 \\
94 \\
65\end{array}$ & $\begin{array}{l}43 \\
43 \\
71 \\
77 \\
55 \\
53 \\
74 \\
58 \\
76 \\
53\end{array}$ & $\begin{array}{l}38 \\
30 \\
57 \\
53 \\
28 \\
50 \\
57 \\
35 \\
55 \\
39\end{array}$ & $\begin{array}{r}27 \\
-1 \\
38 \\
46 \\
7 \\
30 \\
46 \\
28 \\
45 \\
30\end{array}$ & $\begin{array}{r}21 \\
-12 \\
31 \\
27 \\
-3 \\
17 \\
34 \\
13 \\
34 \\
26\end{array}$ & $\begin{array}{l}3.38 \\
4.62 \\
3.01 \\
3.30 \\
3.74 \\
3.56 \\
3.08 \\
3.64 \\
3.01 \\
3.20\end{array}$ \\
\hline $\begin{array}{l}\text { N. H. } \\
\text { G. D. } \\
\text { W. L. } \\
\text { A. B. } \\
\text { F. L. T. } \\
\text { H. H. } \\
\text { B. K. } \\
\text { L. A.S. } \\
\text { M.P. } \\
\text { A. S. }\end{array}$ & $\begin{array}{r}102 \\
98 \\
102 \\
97 \\
92 \\
102 \\
75 \\
92 \\
98 \\
122\end{array}$ & $\begin{array}{l}159 \\
182 \\
151 \\
138 \\
156 \\
159 \\
170 \\
173 \\
149 \\
116\end{array}$ & $\begin{array}{l}118 \\
138 \\
108 \\
109 \\
114 \\
118 \\
129 \\
126 \\
110 \\
80\end{array}$ & $\begin{array}{r}96 \\
113 \\
86 \\
83 \\
76 \\
96 \\
100 \\
86 \\
87 \\
69\end{array}$ & $\begin{array}{l}84 \\
85 \\
54 \\
63 \\
46 \\
84 \\
79 \\
58 \\
70 \\
53\end{array}$ & $\begin{array}{l}72 \\
62 \\
56 \\
52 \\
37 \\
72 \\
58 \\
40 \\
52 \\
42\end{array}$ & $\begin{array}{r}64 \\
40 \\
51 \\
39 \\
0 \\
64 \\
43 \\
0 \\
41 \\
30\end{array}$ & $\begin{array}{r}46 \\
37 \\
31 \\
30 \\
8 \\
46 \\
19 \\
11 \\
23 \\
14\end{array}$ & $\begin{array}{r}34 \\
12 \\
24 \\
21 \\
-18 \\
34 \\
16 \\
-10 \\
20 \\
11\end{array}$ & $\begin{array}{l}3.00 \\
3.36 \\
3.34 \\
3.44 \\
4.62 \\
3.00 \\
3.55 \\
4.62 \\
3.30 \\
3.22\end{array}$ \\
\hline
\end{tabular}

A simple expression for finding the disappearance rate of glucose is to let $C(t)=\frac{1}{2} C(0)$, then on substituting in eq. (1)

$$
\frac{1}{2} \mathrm{C}(0)=\mathrm{C}(0) \mathrm{e}^{-\mathbf{k t}}
$$

or

$$
k=\frac{\log _{e} 2}{t}=\frac{0.693}{t}
$$

\section{ANALYTIC AND GRAPHIC ANALYSIS}

As it is well appreciated in the application of mathematics to practical problems, one deals with inaccurate data. All measurements entering into the calculation involve errors of some magnitude and many of the numerical values used are rounded off. If one plots the observed mgm. per cent of glucose during the rapid glucose tolerance test against time, a parabola is formed. However, assuming that the fasting blood glucose is a constant baseline during the rapid glucose tolerance test, then by plotting $\log _{e}$ (mgm. per cent glucose excess) against time one will get a linear rela-

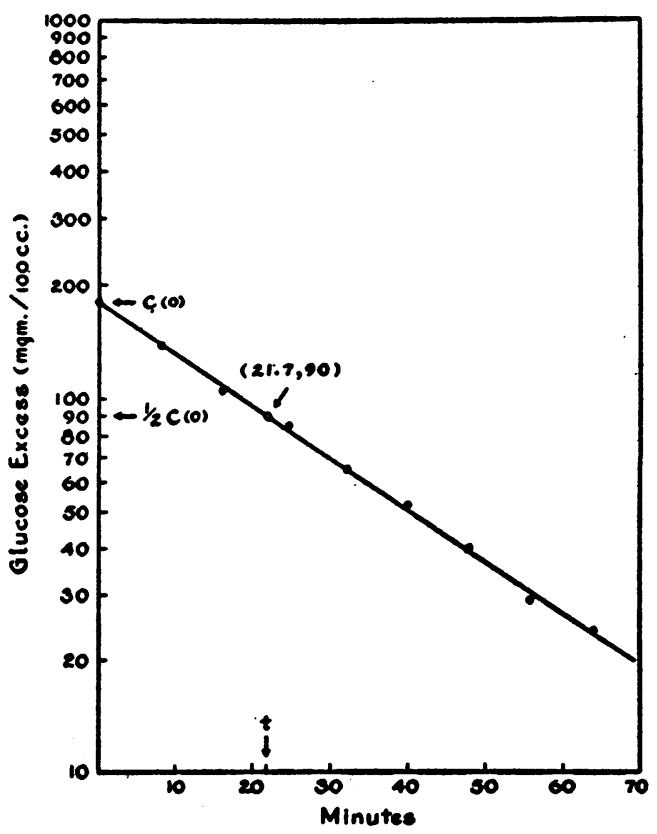

Fig. 2. Glucose Disappearance of Fig. 1 Plotted os Stmilogarithumc Paper-Patient W. B. 
tionship (eq. [1]). By fermentation we found that the non-glucose reducing substances during the rapid glucose tolerance test remained relatively constant (the greatest difference being $5 \mathrm{mgm}$. per cent) in seven normal individuals and six patients with diabetes mellitus. It should be emphasized that any errors inherent in the method, technical or personal, may, at times, be magnified so as not to give a good fit on plotting $\log _{e}$ (mgm. per cent glucose excess) against time. From this graph one can readily find $k$ (the disappearance rate of glucose from the blood) by eq. (2). This is graphically illustrated by plotting glucose excess on the ordinate of semilogarithmic paper against time on the abscissa (Figure 2), where $\mathrm{k}$ is the number of multiples of 2.30 minutes corresponding to each power of 10 through which the glucose excess changes. An analytical and graphical sample is given in Table III.

\section{a. Analytical method}

The graph of the relation between the blood mgm. per cent glucose excess and time is shown in Figure 1. Substituting into eq. (1), one readily

TABLE II

The rapid intravenous glucose tolerance (25 grams) and a repeated (25 grams) test with its rate of removal in normal men

\begin{tabular}{|c|c|c|c|c|c|c|c|c|c|c|c|}
\hline \multirow[b]{2}{*}{ Patient } & & \multirow[b]{2}{*}{$\begin{array}{l}\text { Fasting } \\
\text { glucose }\end{array}$} & \multicolumn{8}{|c|}{ Glucose excess (mgm.\%) } & \multirow{2}{*}{$\begin{array}{l}\text { Rate of } \\
\text { removal } \\
\text { (\%/min.) }\end{array}$} \\
\hline & & & 0 & 8 & 16 & $\stackrel{24}{\text { (time in } m}$ & $\begin{array}{c}32 \\
\text { tes) }\end{array}$ & 40 & 48 & 56 & \\
\hline $\begin{array}{l}\text { R. C. } \\
\text { W. B. } \\
\text { R. C. } \\
\text { M. W. } \\
\text { G. T. }\end{array}$ & $\begin{array}{l}\text { a.* } \\
\text { b. } \\
\text { a. } \\
\text { b. } \\
\text { a. } \\
\text { b. } \\
\text { a. } \\
\text { b. } \\
\text { a. } \\
\text { b. }\end{array}$ & $\begin{array}{r}83 \\
91 \\
85 \\
80 \\
97 \\
105 \\
85 \\
117 \\
93 \\
80\end{array}$ & $\begin{array}{l}183 \\
177 \\
177 \\
165 \\
183 \\
184 \\
157 \\
118 \\
179 \\
230\end{array}$ & $\begin{array}{r}124 \\
130 \\
138 \\
123 \\
141 \\
147 \\
112 \\
80 \\
140 \\
160\end{array}$ & $\begin{array}{r}95 \\
82 \\
105 \\
103 \\
109 \\
104 \\
82 \\
70 \\
101 \\
128\end{array}$ & $\begin{array}{l}67 \\
57 \\
85 \\
84 \\
82 \\
79 \\
67 \\
55 \\
81 \\
93\end{array}$ & $\begin{array}{l}51 \\
44 \\
65 \\
67 \\
65 \\
68 \\
56 \\
43 \\
59 \\
72\end{array}$ & $\begin{array}{l}42 \\
35 \\
52 \\
50 \\
53 \\
48 \\
48 \\
38 \\
50 \\
54\end{array}$ & $\begin{array}{l}35 \\
21 \\
40 \\
38 \\
41 \\
38 \\
36 \\
23 \\
35 \\
33\end{array}$ & $\begin{array}{l}19 \\
14 \\
29 \\
23 \\
26 \\
15 \\
18 \\
17 \\
19 \\
29\end{array}$ & $\begin{array}{l}3.90 \\
4.06 \\
3.20 \\
3.20 \\
3.20 \\
3.28 \\
3.20 \\
3.36 \\
3.59 \\
3.60\end{array}$ \\
\hline $\begin{array}{l}\text { Dr. J. } \\
\text { K. H. } \\
\text { E. R. } \\
\text { L. E. } \\
\text { F. Mc. }\end{array}$ & $\begin{array}{l}\text { a. } \\
\text { b. } \\
\text { a. } \\
\text { b. } \\
\text { a. } \\
\text { b. } \\
\text { a. } \\
\text { b. } \\
\text { a. } \\
\text { b. }\end{array}$ & $\begin{array}{r}95 \\
80 \\
128 \\
128 \\
80 \\
100 \\
92 \\
90 \\
92 \\
87\end{array}$ & $\begin{array}{l}182 \\
176 \\
190 \\
172 \\
191 \\
187 \\
188 \\
216 \\
183 \\
203\end{array}$ & $\begin{array}{l}152 \\
148 \\
132 \\
120 \\
165 \\
143 \\
143 \\
160 \\
125 \\
147\end{array}$ & $\begin{array}{r}98 \\
107 \\
124 \\
87 \\
118 \\
102 \\
105 \\
135 \\
91 \\
103\end{array}$ & $\begin{array}{r}81 \\
80 \\
89 \\
59 \\
95 \\
86 \\
85 \\
105 \\
62 \\
61\end{array}$ & $\begin{array}{l}70 \\
70 \\
57 \\
46 \\
75 \\
62 \\
69 \\
83 \\
41 \\
55\end{array}$ & $\begin{array}{l}50 \\
55 \\
46 \\
16 \\
58 \\
48 \\
52 \\
66 \\
26 \\
30\end{array}$ & $\begin{array}{r}38 \\
47 \\
34 \\
8 \\
48 \\
29 \\
36 \\
53 \\
18 \\
15\end{array}$ & $\begin{array}{r}17 \\
23 \\
25 \\
8 \\
36 \\
21 \\
29 \\
38 \\
8 \\
6\end{array}$ & $\begin{array}{l}3.08 \\
2.97 \\
3.98 \\
4.06 \\
3.24 \\
3.35 \\
3.20 \\
3.20 \\
4.84 \\
4.92\end{array}$ \\
\hline $\begin{array}{l}\text { C. A. } \\
\text { H. H. } \\
\text { H. N. } \\
\text { B. D. } \\
\text { F. R. }\end{array}$ & $\begin{array}{l}\text { a. } \\
\text { b. } \\
\text { a. } \\
\text { b. } \\
\text { a. } \\
\text { b. } \\
\text { a. } \\
\text { b. } \\
\text { a. } \\
\text { b. }\end{array}$ & $\begin{array}{r}83 \\
83 \\
95 \\
89 \\
97 \\
96 \\
97 \\
102 \\
91 \\
85\end{array}$ & $\begin{array}{l}204 \\
207 \\
165 \\
180 \\
158 \\
200 \\
167 \\
140 \\
230 \\
160\end{array}$ & $\begin{array}{r}141 \\
147 \\
118 \\
137 \\
131 \\
150 \\
116 \\
93 \\
150 \\
139\end{array}$ & $\begin{array}{r}94 \\
108 \\
95 \\
107 \\
106 \\
109 \\
88 \\
74 \\
124 \\
103\end{array}$ & $\begin{array}{r}75 \\
72 \\
70 \\
76 \\
86 \\
95 \\
71 \\
48 \\
114 \\
81\end{array}$ & $\begin{array}{l}59 \\
65 \\
51 \\
55 \\
73 \\
82 \\
52 \\
29 \\
84 \\
58\end{array}$ & $\begin{array}{l}34 \\
36 \\
37 \\
47 \\
49 \\
71 \\
28 \\
27 \\
67 \\
45\end{array}$ & $\begin{array}{l}28 \\
19 \\
29 \\
38 \\
36 \\
64 \\
28 \\
19 \\
57 \\
36\end{array}$ & $\begin{array}{r}10 \\
7 \\
11 \\
11 \\
28 \\
55 \\
1 \\
4 \\
36 \\
28\end{array}$ & $\begin{array}{l}4.16 \\
4.21 \\
3.67 \\
3.67 \\
3.04 \\
3.00 \\
3.95 \\
3.98 \\
3.35 \\
3.43\end{array}$ \\
\hline $\begin{array}{l}\text { G. F. E. } \\
\text { A. Z. } \\
\text { E. G. } \\
\text { R. C. H. } \\
\text { W. W. }\end{array}$ & $\begin{array}{l}\text { a. } \\
\text { b. } \\
\text { a. } \\
\text { b. } \\
\text { a. } \\
\text { b. } \\
\text { a. } \\
\text { b. } \\
\text { a. } \\
\text { b. }\end{array}$ & $\begin{array}{l}95 \\
83 \\
78 \\
83 \\
85 \\
93 \\
87 \\
92 \\
98 \\
90\end{array}$ & $\begin{array}{l}138 \\
147 \\
196 \\
164 \\
187 \\
167 \\
185 \\
138 \\
192 \\
186\end{array}$ & $\begin{array}{r}99 \\
105 \\
142 \\
125 \\
143 \\
119 \\
134 \\
102 \\
139 \\
135\end{array}$ & $\begin{array}{r}77 \\
83 \\
117 \\
95 \\
97 \\
80 \\
95 \\
67 \\
107 \\
115\end{array}$ & $\begin{array}{l}57 \\
57 \\
90 \\
75 \\
63 \\
46 \\
74 \\
45 \\
87 \\
95\end{array}$ & $\begin{array}{l}43 \\
45 \\
77 \\
59 \\
33 \\
36 \\
48 \\
33 \\
64 \\
74\end{array}$ & $\begin{array}{r}38 \\
43 \\
53 \\
48 \\
9 \\
-5 \\
35 \\
14 \\
50 \\
53\end{array}$ & $\begin{array}{r}27 \\
26 \\
46 \\
42 \\
-1 \\
-18 \\
17 \\
3 \\
25 \\
40\end{array}$ & $\begin{array}{r}21 \\
20 \\
27 \\
28 \\
-12 \\
-20 \\
5 \\
-11 \\
9 \\
23\end{array}$ & $\begin{array}{l}3.38 \\
3.41 \\
3.30 \\
3.31 \\
4.62 \\
4.62 \\
4.53 \\
4.62 \\
3.36 \\
3.36\end{array}$ \\
\hline
\end{tabular}

* a. 25 grams of Glucose.

b. 25 grams of Glucose. 
TABLE III

Observed blood glucose values of $W$. B., with the four minute value set at time zero

\begin{tabular}{|c|c|c|c|c|c|c|c|c|c|c|}
\hline$\underset{\text { (minutes) }}{\text { Time }}$ & Fasting & 4 & 12 & 20 & 28 & 36 & 44 & 52 & 60 & 68 \\
\hline $\begin{array}{l}\text { Obeerved } \\
\text { glucose } \\
\text { (mgm.\%) }\end{array}$ & 85 & 265 & 223 & 190 & 170 & 150 & 137 & 125 & 114 & 109 \\
\hline $\begin{array}{l}\text { Time } \\
\text { (4 minutes }\end{array}$ & & O & 8 & 16 & 24 & 32 & 40 & 48 & 56 & 64 \\
\hline $\begin{array}{l}\text { Glucose excess } \\
\text { (mgm.\%) }\end{array}$ & & 180 & 138 & 105 & 85 & 65 & 52 & 40 & 29 & 24 \\
\hline
\end{tabular}

finds the $k$, the disappearance rate of glucose from the blood for two points.

$$
\mathrm{k}=\frac{2.3 \log _{10} 180 / 65}{32-0}=0.0319
$$

or

$$
k=3.19 \text { per cent per minute. }
$$

\section{b. Graphic method}

The semilogarithmic graph of the relation between the blood glucose excess and time is illustrated in Figure 2.
By using the relationship shown in eq. (2) where $C(t)$ equals $\frac{1}{2} C(0)$, to find $t$ from Figure 2 we see that $\frac{1}{2} \mathrm{C}(\mathrm{o})$ equals $90 \mathrm{mgm}$. per $100 \mathrm{cc}$. with a corresponding $t$ equal to 21.7 minutes. Substituting into eq. (2), one finds the average disappearance rate of glucose.

$$
\mathrm{k}=\frac{0.693}{21.7}=0.0319
$$

or

$$
k=3.19 \text { per cent per minute. }
$$

The skill required for drawing an accurate freehand curve is not required with the graphic method and the average $\mathrm{k}$ for all points is more readily and easily obtained.

It appears that the disappearance rate of glucose from the blood is apparently the resultant of a number of simultaneous processes that best fit a simple unimolecular equation. The disappearance rate of glucose in 70 normal men with no family history of diabetes mellitus who received 25 grams

\begin{tabular}{|c|c|c|c|c|c|c|c|c|c|c|c|c|c|}
\hline \multirow[b]{2}{*}{ Patient } & & \multirow[b]{2}{*}{$\begin{array}{l}\text { Fasting } \\
\text { glucose }\end{array}$} & \multicolumn{10}{|c|}{ Glucose excess (mgm.\%) } & \multirow{2}{*}{$\begin{array}{l}\text { Rate of } \\
\text { removal } \\
\text { (\%/min.) }\end{array}$} \\
\hline & & & $\mathbf{0}$ & 8 & 16 & 24 & 32 & $\begin{array}{l}40 \\
\text { ttes) }\end{array}$ & 48 & 56 & 64 & 72 & \\
\hline $\begin{array}{l}\text { R. C. } \\
\text { G. T. } \\
\text { Dr. J. } \\
\text { L. E. } \\
\text { F. Mc. }\end{array}$ & $\begin{array}{l}\text { a.* } \\
\text { b. } \\
\text { a. } \\
\text { b. } \\
\text { a. } \\
\text { b. } \\
\text { a. } \\
\text { b. } \\
\text { a. } \\
\text { b. }\end{array}$ & $\begin{array}{r}105 \\
96 \\
93 \\
84 \\
80 \\
92 \\
92 \\
96 \\
87 \\
96\end{array}$ & $\begin{array}{l}184 \\
249 \\
179 \\
311 \\
176 \\
256 \\
188 \\
302 \\
203 \\
214\end{array}$ & $\begin{array}{l}149 \\
200 \\
140 \\
228 \\
148 \\
192 \\
143 \\
209 \\
147 \\
166\end{array}$ & $\begin{array}{l}103 \\
143 \\
101 \\
191 \\
107 \\
171 \\
105 \\
161 \\
103 \\
114\end{array}$ & $\begin{array}{r}79 \\
112 \\
81 \\
140 \\
80 \\
143 \\
85 \\
126 \\
61 \\
69\end{array}$ & $\begin{array}{r}68 \\
87 \\
59 \\
109 \\
70 \\
118 \\
69 \\
94 \\
55 \\
46\end{array}$ & $\begin{array}{l}48 \\
74 \\
50 \\
89 \\
55 \\
95 \\
52 \\
79 \\
30 \\
47\end{array}$ & $\begin{array}{l}38 \\
35 \\
35 \\
69 \\
47 \\
75 \\
36 \\
49 \\
15 \\
24\end{array}$ & $\begin{array}{r}15 \\
15 \\
19 \\
49 \\
23 \\
75 \\
29 \\
32 \\
6 \\
16\end{array}$ & $\begin{array}{r}15 \\
-6 \\
8 \\
35 \\
6 \\
46 \\
16 \\
16 \\
6 \\
10\end{array}$ & $\begin{array}{r}10 \\
-1 \\
0 \\
14 \\
0 \\
34 \\
4 \\
-5 \\
-2 \\
2\end{array}$ & $\begin{array}{l}3.28 \\
3.28 \\
3.59 \\
3.51 \\
2.97 \\
2.97 \\
3.20 \\
3.20 \\
4.92 \\
4.71\end{array}$ \\
\hline $\begin{array}{l}\text { E. S. } \\
\text { R. S. } \\
\text { H. H. } \\
\text { K. N. } \\
\text { J. S. }\end{array}$ & $\begin{array}{l}\text { a. } \\
\text { b. } \\
\text { a. } \\
\text { b. } \\
\text { a. } \\
\text { b. } \\
\text { a. } \\
\text { b. } \\
\text { a. } \\
\text { b. }\end{array}$ & $\begin{array}{l}95 \\
90 \\
88 \\
88 \\
89 \\
77 \\
96 \\
87 \\
87 \\
98\end{array}$ & $\begin{array}{l}178 \\
238 \\
150 \\
204 \\
180 \\
227 \\
200 \\
198 \\
164 \\
197\end{array}$ & $\begin{array}{l}140 \\
188 \\
111 \\
165 \\
137 \\
184 \\
150 \\
170 \\
135 \\
148\end{array}$ & $\begin{array}{r}107 \\
172 \\
92 \\
130 \\
107 \\
144 \\
109 \\
138 \\
110 \\
115\end{array}$ & $\begin{array}{r}87 \\
140 \\
72 \\
120 \\
76 \\
109 \\
95 \\
124 \\
82 \\
91\end{array}$ & $\begin{array}{r}68 \\
120 \\
59 \\
92 \\
55 \\
85 \\
82 \\
105 \\
61 \\
59\end{array}$ & $\begin{array}{l}43 \\
98 \\
46 \\
72 \\
47 \\
56 \\
71 \\
83 \\
42 \\
46\end{array}$ & $\begin{array}{l}42 \\
84 \\
13 \\
55 \\
38 \\
45 \\
64 \\
61 \\
35 \\
29\end{array}$ & $\begin{array}{l}23 \\
73 \\
25 \\
37 \\
11 \\
30 \\
55 \\
49 \\
23 \\
18\end{array}$ & $\begin{array}{r}17 \\
55 \\
17 \\
32 \\
3 \\
19 \\
41 \\
38 \\
8 \\
17\end{array}$ & $\begin{array}{r}0 \\
34 \\
7 \\
12 \\
-4 \\
8 \\
32 \\
38 \\
3 \\
9\end{array}$ & $\begin{array}{l}3.04 \\
3.12 \\
3.12 \\
3.05 \\
\mathbf{3 . 6 7} \\
\mathbf{3 . 6 7} \\
\mathbf{3 . 0 0} \\
\mathbf{3 . 0 0} \\
\mathbf{3 . 8 2} \\
\mathbf{3 . 9 0}\end{array}$ \\
\hline $\begin{array}{l}\text { B. D. } \\
\text { R. F. } \\
\text { R. C. H. }\end{array}$ & $\begin{array}{l}\text { a. } \\
\text { b. } \\
\text { a. } \\
\text { b. } \\
\text { a. } \\
\text { b. }\end{array}$ & $\begin{array}{r}102 \\
105 \\
87 \\
100 \\
87 \\
91\end{array}$ & $\begin{array}{l}140 \\
245 \\
206 \\
230 \\
185 \\
230\end{array}$ & $\begin{array}{r}93 \\
177 \\
143 \\
150 \\
134 \\
147\end{array}$ & $\begin{array}{r}74 \\
149 \\
101 \\
107 \\
95 \\
109\end{array}$ & $\begin{array}{r}48 \\
120 \\
85 \\
73 \\
74 \\
87\end{array}$ & $\begin{array}{r}29 \\
100 \\
54 \\
46 \\
48 \\
71\end{array}$ & $\begin{array}{l}14 \\
74 \\
40 \\
22 \\
35 \\
54\end{array}$ & $\begin{array}{l}19 \\
48 \\
28 \\
22 \\
17 \\
25\end{array}$ & $\begin{array}{r}4 \\
35 \\
10 \\
-10 \\
5 \\
13\end{array}$ & $\begin{array}{r}-4 \\
20 \\
4 \\
-27 \\
-4 \\
0\end{array}$ & $\begin{array}{r}-12 \\
11 \\
-10 \\
-22 \\
-5 \\
0\end{array}$ & $\begin{array}{l}3.98 \\
3.98 \\
4.30 \\
4.60 \\
4.21 \\
4.21\end{array}$ \\
\hline
\end{tabular}
of glucose intravenously was found to range from

TABLE IV

The removal rate on comparing the 25 grams and 35 grams rapid intravenous glucose tolerance in normal men

* a. 25 grams of Glucose.

b. 35 grams of Glucose. 
TABLE $V$

The rapid intravenous glucose (25 grams) tolerance test in diabetes mellitus

\begin{tabular}{|c|c|c|c|c|c|c|c|c|c|c|c|c|}
\hline \multirow[b]{2}{*}{ Patient } & \multirow[b]{2}{*}{ Fasting } & \multicolumn{10}{|c|}{ Glucose excess (mgm.\%) } & \multirow{2}{*}{$\begin{array}{c}\text { Rate of } \\
\text { removal } \\
(\% / \mathrm{min} .)\end{array}$} \\
\hline & & $\mathbf{0}$ & 8 & 16 & 24 & $\begin{array}{c}32 \\
\text { (time in }\end{array}$ & $\begin{array}{c}40 \\
\text { inutes) }\end{array}$ & 48 & 56 & 64 & 72 & \\
\hline \multicolumn{13}{|c|}{ Very mild diabetes mellitus } \\
\hline $\begin{array}{l}\text { O. D. } \\
\text { W. } \\
\text { E. J. } \\
\text { R. S. } \\
\text { L. L. } \\
\text { A. R. } \\
\text { M. } \\
\text { O. T. } \\
\text { D. H. } \\
\text { W. G. }\end{array}$ & $\begin{array}{r}105 \\
94 \\
95 \\
147 \\
146 \\
160 \\
115 \\
98 \\
105 \\
97\end{array}$ & $\begin{array}{l}187 \\
149 \\
170 \\
106 \\
174 \\
236 \\
153 \\
202 \\
130 \\
213\end{array}$ & $\begin{array}{r}168 \\
134 \\
138 \\
99 \\
136 \\
172 \\
138 \\
157 \\
105 \\
155\end{array}$ & $\begin{array}{r}142 \\
109 \\
119 \\
89 \\
110 \\
156 \\
105 \\
139 \\
90 \\
128\end{array}$ & $\begin{array}{r}123 \\
100 \\
108 \\
78 \\
102 \\
130 \\
98 \\
121 \\
75 \\
114\end{array}$ & $\begin{array}{r}105 \\
88 \\
90 \\
71 \\
78 \\
116 \\
85 \\
112 \\
67 \\
98\end{array}$ & $\begin{array}{r}90 \\
81 \\
83 \\
61 \\
68 \\
100 \\
79 \\
102 \\
60 \\
93\end{array}$ & $\begin{array}{l}71 \\
66 \\
73 \\
60 \\
48 \\
94 \\
79 \\
65 \\
52 \\
89\end{array}$ & $\begin{array}{l}63 \\
48 \\
53 \\
57 \\
44 \\
72 \\
46 \\
58 \\
45 \\
54\end{array}$ & $\begin{array}{l}38 \\
42 \\
48 \\
43 \\
38 \\
64 \\
28 \\
45 \\
38 \\
40\end{array}$ & $\begin{array}{l}38 \\
19 \\
37 \\
48 \\
30 \\
70 \\
35 \\
47 \\
37 \\
34\end{array}$ & $\begin{array}{l}2.03 \\
1.64 \\
1.79 \\
1.21 \\
2.46 \\
1.83 \\
2.10 \\
2.27 \\
1.83 \\
2.42\end{array}$ \\
\hline $\begin{array}{l}\text { M. B. } \\
\text { O. } \\
\text { B. } \\
\text { A. P. } \\
\text { W. S. } \\
\text { C. F. } \\
\text { W. W. } \\
\text { A. K. } \\
\text { E. C. } \\
\text { W. W. }\end{array}$ & $\begin{array}{r}94 \\
80 \\
94 \\
102 \\
135 \\
95 \\
115 \\
158 \\
145 \\
143\end{array}$ & $\begin{array}{l}179 \\
210 \\
153 \\
134 \\
135 \\
175 \\
125 \\
122 \\
154 \\
119\end{array}$ & $\begin{array}{l}134 \\
153 \\
124 \\
113 \\
110 \\
132 \\
116 \\
100 \\
125 \\
106\end{array}$ & $\begin{array}{r}117 \\
138 \\
111 \\
93 \\
93 \\
121 \\
102 \\
75 \\
99 \\
101\end{array}$ & $\begin{array}{r}100 \\
118 \\
100 \\
83 \\
68 \\
113 \\
87 \\
64 \\
85 \\
90\end{array}$ & $\begin{array}{r}88 \\
105 \\
92 \\
78 \\
65 \\
85 \\
75 \\
52 \\
72 \\
79\end{array}$ & $\begin{array}{l}73 \\
95 \\
78 \\
65 \\
59 \\
71 \\
68 \\
43 \\
60 \\
81\end{array}$ & $\begin{array}{l}66 \\
77 \\
71 \\
58 \\
51 \\
63 \\
64 \\
31 \\
49 \\
72\end{array}$ & $\begin{array}{l}52 \\
56 \\
56 \\
45 \\
40 \\
45 \\
62 \\
28 \\
40 \\
71\end{array}$ & $\begin{array}{l}42 \\
35 \\
43 \\
28 \\
20 \\
33 \\
56 \\
20 \\
35 \\
65\end{array}$ & $\begin{array}{l}31 \\
31 \\
31 \\
23 \\
30 \\
16 \\
56 \\
19 \\
27 \\
56\end{array}$ & $\begin{array}{l}2.22 \\
2.18 \\
1.79 \\
1.87 \\
2.07 \\
2.42 \\
1.26 \\
2.19 \\
2.32 \\
0.93\end{array}$ \\
\hline $\begin{array}{l}\text { J. E. } \\
\text { J. L. } \\
\text { R. S. } \\
\text { B. O. } \\
\text { E. F. } \\
\text { F. S. }\end{array}$ & $\begin{array}{r}111 \\
113 \\
104 \\
134 \\
104 \\
89\end{array}$ & $\begin{array}{l}143 \\
142 \\
148 \\
131 \\
149 \\
125\end{array}$ & $\begin{array}{l}131 \\
130 \\
124 \\
120 \\
143 \\
109\end{array}$ & $\begin{array}{r}119 \\
112 \\
103 \\
114 \\
126 \\
99\end{array}$ & $\begin{array}{r}107 \\
97 \\
84 \\
99 \\
126 \\
83\end{array}$ & $\begin{array}{r}99 \\
82 \\
76 \\
99 \\
109 \\
78\end{array}$ & $\begin{array}{r}90 \\
76 \\
60 \\
88 \\
110 \\
73\end{array}$ & $\begin{array}{l}83 \\
65 \\
47 \\
83 \\
99 \\
67\end{array}$ & $\begin{array}{l}81 \\
55 \\
44 \\
76 \\
91 \\
61\end{array}$ & $\begin{array}{l}72 \\
45 \\
36 \\
69 \\
74 \\
55\end{array}$ & $\begin{array}{l}66 \\
36 \\
29 \\
62 \\
78 \\
53\end{array}$ & $\begin{array}{l}1.09 \\
1.77 \\
2.35 \\
0.99 \\
0.93 \\
1.23\end{array}$ \\
\hline \multicolumn{13}{|c|}{ Severe diabetes mellitus } \\
\hline $\begin{array}{l}\text { J. U. } \\
\text { G. E. } \\
\text { M. C. } \\
\text { E. J. } \\
\text { B. P. } \\
\text { A. N. } \\
\text { E. W. } \\
\text { F. D. } \\
\text { A. D. } \\
\text { W. H. }\end{array}$ & $\begin{array}{l}206 \\
322 \\
136 \\
224 \\
155 \\
155 \\
198 \\
228 \\
192 \\
168\end{array}$ & $\begin{array}{r}210 \\
152 \\
200 \\
196 \\
161 \\
133 \\
65 \\
90 \\
112 \\
222\end{array}$ & $\begin{array}{r}160 \\
116 \\
154 \\
136 \\
152 \\
99 \\
73 \\
72 \\
100 \\
176\end{array}$ & $\begin{array}{r}124 \\
114 \\
124 \\
108 \\
146 \\
97 \\
70 \\
64 \\
92 \\
158\end{array}$ & $\begin{array}{r}116 \\
102 \\
124 \\
96 \\
132 \\
90 \\
67 \\
64 \\
81 \\
146\end{array}$ & $\begin{array}{r}110 \\
104 \\
104 \\
84 \\
122 \\
75 \\
64 \\
56 \\
76 \\
132\end{array}$ & $\begin{array}{r}106 \\
78 \\
104 \\
80 \\
108 \\
77 \\
62 \\
52 \\
69 \\
136\end{array}$ & $\begin{array}{r}84 \\
76 \\
104 \\
76 \\
109 \\
66 \\
65 \\
47 \\
63 \\
102\end{array}$ & $\begin{array}{r}70 \\
74 \\
82 \\
50 \\
102 \\
59 \\
65 \\
43 \\
61 \\
92\end{array}$ & $\begin{array}{r}62 \\
66 \\
82 \\
48 \\
102 \\
54 \\
62 \\
39 \\
58 \\
82\end{array}$ & $\begin{array}{l}50 \\
60 \\
82 \\
46 \\
87 \\
57 \\
64 \\
39 \\
56 \\
80\end{array}$ & $\begin{array}{l}1.64 \\
1.13 \\
1.09 \\
1.87 \\
0.85 \\
1.12 \\
0.23 \\
0.97 \\
1.06 \\
1.48\end{array}$ \\
\hline $\begin{array}{l}\text { O. N. } \\
\text { A. B. } \\
\text { A. D. }\end{array}$ & $\begin{array}{l}192 \\
262 \\
168\end{array}$ & $\begin{array}{r}64 \\
76 \\
110\end{array}$ & $\begin{array}{r}62 \\
70 \\
110\end{array}$ & $\begin{array}{r}58 \\
62 \\
100\end{array}$ & $\begin{array}{l}57 \\
58 \\
92\end{array}$ & $\begin{array}{l}61 \\
49 \\
85\end{array}$ & $\begin{array}{l}63 \\
45 \\
79\end{array}$ & $\begin{array}{l}61 \\
46 \\
75\end{array}$ & $\begin{array}{l}57 \\
43 \\
74\end{array}$ & $\begin{array}{l}57 \\
36 \\
66\end{array}$ & $\begin{array}{l}55 \\
30 \\
52\end{array}$ & $\begin{array}{l}0.15 \\
1.23 \\
0.85\end{array}$ \\
\hline
\end{tabular}

3.00 to 4.84 per cent per minute (Table I). The test was repeated with 25 grams of glucose one to three months later on 20 normal subjects who had a dietary intake of at least 150 grams of carbohydrate daily. The disappearance rate of glucose (k) was constant for each subject (Table II), i.e., the disappearance rate was reproducible.

In order to study the effect of a larger dose the test was repeated with 35 grams of glucose in 13 normal individuals who had been previously studied with the 25 grams dose and who had a dietary intake of at least 150 grams of carbohydrate daily (Table IV). It was found that the disappearance rate was unaltered by the larger dosage, i.e., it was constant for the individual tested. Figure 3 illustrates the observed data of the two dosage tests in subject W. B. plotted on semilogarithmic graph paper. It is obvious that the slopes of the curves, and therefore the removal rates, are the same and are unaltered by this change in dosage. It should also be noted that an apparent oscillation of the curve is exaggerated with the $\mathbf{3 5}$ grams dose but 


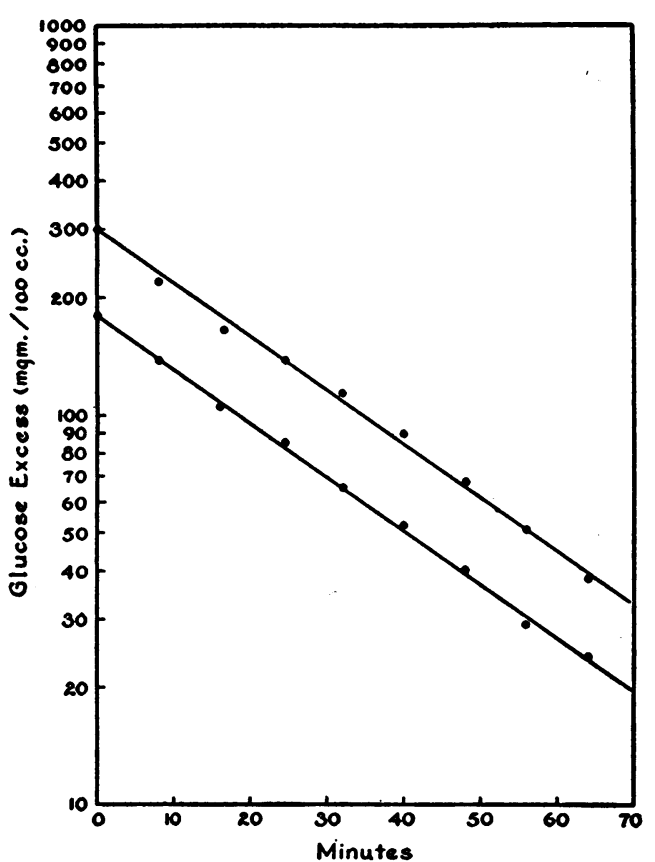

Fig. 3. Comparison of the Glucose Disappearance after 25 and 35 Gram Test of Glucose-Patient W. B.

that the values of the disappearance rate of glucose following dosages of 25 and 35 grams are the same. The apparent oscillations may be related to numerous simultaneous processes in the body.

A group of 26 patients with known mild diabetes controlled by diet (the majority had normal fasting blood sugar values) was similarly studied with the $\mathbf{2 5}$ grams test. The disappearance rate of glucose was found to range from 0.93 to 2.46 per cent per minute (Table V). This difference is obviously highly significant on comparison with the normal series. A distinct, sharp separation of the mild diabetic patients from the normal subjects is readily apparent. Also studied were 13 patients with severe diabetes who were found to have a lower range ( 0.23 to 1.64 per cent per minute) for the rate of removal of glucose (Table V).

The mean quantity of glucose lost in the urine during the test varied from 0.2 to 1.0 gram in 22 normal men, and from 0.7 to 2.9 grams in 19 mild and severe diabetics.

\section{DISCUSSION}

The first attempt to analyze the rapid glucose tolerance curve was by Jörgenson (4) (1926) and
Ross (5) (1938) who measured the area under the observed curve to interpret their data. Fishberg (6) (1930) found that the rate of disappearance of foreign sugars injected into the blood stream obeyed a monomolecular reaction velocity equation. An excellent detailed treatment for determining the intravenous glucose tolerance equation was shown by Greville (7).

The fate of the intravenously administered glucose has not been definitely determined. Loss of glucose in the urine is not an important factor since only a small amount was found in the patients tested ( 0.2 to 2 grams). The disappearance of glucose from the blood merely by diffusion into the tissues is unlikely because: (1) the disappearance rate of glucose from the blood in mild diabetics and in normal individuals with a similar fasting blood sugar level showed a distinct difference, and (2) the disappearance rate did not vary with the dose of glucose. It may be suggested that changes in blood volume resulting from the injected glucose may produce an apparent loss from the blood stream. However, Pijoan and Gibson (8) using the Evans blue dye method found that there was no significant change in blood volume after the injection of 25 grams of glucose in $55 \mathrm{cc}$. of water.

The ferricyanide reaction as a method of determining blood glucose was introduced by Hagedorn and Jensen $(9,10)$. For routine purposes the disadvantage of this procedure was that it measured all reducing substances in the blood in addition to glucose. Measurement of the mgm. per cent glucose excess in blood, determined by the difference in reducing power before and after the injection of glucose, avoids consideration of the non-glucose-reducing substances and thus, for this purpose, the ferricyanide procedure becomes the method of choice.

The variation in the disappearance rate of glucose in normal individuals could not be correlated with the height, weight (i.e., non-obese patients), age or dose administered. It was apparent that the disappearance rate was reproducible for the individual regardless of dose. An apparent oscillation of the plotted curves was noted with the 35 grams injection. By the rapid glucose tolerance test, one can obtain an index of the disappearance rate of glucose from the blood. With values of glucose excess below $30 \mathrm{mgm}$. per 100 cc. the plotted curve tends to become distorted. 
The above interpretation has some advantages in that (1) the disappearance rate of glucose in normals is constant, reproducible, and independent of dosage for each individual, (2) there is a marked separation of the normal from known borderline diabetes mellitus, (3) a precise manner of interpreting glucose tolerance curves is possible, (4) a rapid glucose tolerance test may be completed within 60 minutes, and (5) the disappearance rate of glucose from the blood appears to fit best a simple expression (eq. [1]).

\section{SUMMARY}

A study has been made of the rapid intravenous glucose tolerance test by following the disappearance rate of glucose. It was found that the rate of removal in the normal individual maintained on an adequate carbohydrate diet remained constant on repeated tests and was independent of the dose administered for each subject.

By determining the removal rate, a sharp separation was found between the patients with known mild diabetes (26), severe diabetes (13), and the group of normal controls (70). A decreased rate of removal has been observed in other conditions, including obesity unrelated to a family history of diabetes, inflammatory disease, uremia, weight loss due to diet, and decompensated portal cirrhosis.

\section{REFERENCES}

1. Tunbridge, R. E., and Allibone, E. C., The intravenous dextrose tolerance test. Quart. J. Med., 1940, 9, 11.

2. Horvath, S. M., and Knehr, C. A., Adaptation of the Folin-Malmros micro blood sugar method to photoelectric colorimeter. J. Biol. Chem., 1941, 140, 869.

3. Folin, O., and Malmros, H., An improved form of Folin's micro method for blood sugar determinations. J. Biol. Chem., 1929, 83, 115.

4. Jörgensen, S., Comparison between the intravenous and oral application of glucose for loading of the carbohydrate-metabolism. Acta med. Scandinav., 1926-27, 65, 116.

5. Ross, C. W., with the technical assistance of Tonks, E. L., The determination of glucose tolerance. Arch. Dis. Childhood, 1938, 13, 289.

6. Fishberg, E. H., The rate of disappearance of foreign sugar from the blood stream. J. Biol. Chem., 1930, 86, 665 .

7. Greville, G. D., The intravenous glucose tolerance equation. Biochem. J., 1943, 37, 17.

8. Pijoan, M., and Gibson, J. G., II, The rate of disappearance of intravenously administered dextrose in the human subject. Am. J. Physiol., 1938, 121, 534.

9. Hagedorn, H. C., and Jensen, B. N., Zur Mikrobestimmung des Blutzuckers mittels Ferricyanid. Biochem. Z., 1923, 135, 46.

10. Hagedorn, H. D., and Jensen, B. N., Die Ferricyanidmethode zur Blutzuckerbestimmung II. Biochem. Z., 1923, 137, 92. 